\title{
PROGRAMACIÓN POR OBJETIVOS PARA EL DIMENSIONAMIENTO Y LA ASIGNACIÓN DE UNA FLOTA DE CAMIONES EN UNA EMPRESA MINERA
}

\author{
Edmundo Quiroz-Silva \\ edmundoquiroz@belcorp.biz \\ Universidad de Lima. Lima, Perú
}

La toma de decisiones en la minería es un proceso complicado. Las decisiones con respecto al dimensionamiento y a la asignación de la flota de camiones a diferentes minas, en consideración con el cumplimiento de la producción y del costo total objetivo, son de suma importancia para las empresas mineras, ya que esto afecta directamente las utilidades que pueden obtener. Este documento presenta un modelo de programación por objetivos (con base en la programación lineal) para optimizar las operaciones mineras de extracción en minas socavón, tomando en consideración tres objetivos: (a) Minimizar las desviaciones de la producción objetivo de diferentes minerales, (b) minimizar el excedente del costo operativo total designado y (c) maximizar el uso de la capacidad máxima de las plantas concentradores. El modelo determina la cantidad de camiones necesarios y proporciona la asignación óptima a cada mina, mientras se abordan los objetivos y las limitaciones de la operación minera; el modelo planteado es luego validado utilizando datos reales de una empresa minera peruana.

Palabras clave: asignación de camiones, minería, programación lineal, programación por objetivos

\section{Abstract}

\section{Goal programming for sizing and allocation of a fleet of trucks in a mining company}

Decision making in mining is a complicated process. Decisions regarding the sizing and allocation of the fleet of trucks to different mines, considering the compliance with production and the total objective cost, are of paramount importance to mining companies as this directly affects their profits. This document presents a model of goal programming (based on linear programming) to optimize mining operations in underground mining taking into account three goals: (a) To minimize deviations from the target production of different minerals, (b) to minimize the surplus of the total designated operating cost and (c) to maximize the use of concentrator plants' maximum capacity. The model determines the number of trucks needed and provides the optimal allocation to each mine while addressing the goals and limitations of the mining operation. The proposed model was validated using real data from a Peruvian mining company.

Keywords: truck allocation, mining, linear programming, goal programming 


\section{Introducción}

El sector minero es, en principio, un negocio de costos, donde el producto final se controla con los precios que dicta el mercado (basados en la oferta y la demanda mundial), sobre el cual ninguna entidad tiene control directo. Sin embargo, donde las empresas sí tienen control es sobre los costos propios de la operación (perforación, extracción, transporte y tratamiento del mineral), dependiendo de un adecuado control de los mismos será el margen de ganancias que alcancen. Esta es razón suficiente para que las empresas mineras estén atentas a obtener una adecuada optimización de costos, sobre todo en las épocas donde el precio de los metales baja considerablemente. En la industria minera, el dimensionamiento y la asignación de la flota de camiones en las operaciones de extracción y tratamiento de los minerales es un proceso crítico; una correcta asignación de camiones genera ahorros evidentes. Sin embargo, encontrar la solución ante esta clase de problema no es sencillo, pues engloba un gran número de variables. Muchas veces el enfoque de respuesta que se da no es el adecuado, lo cual genera sobrecostos y errores. El uso de modelos matemáticos es necesario para darle frente a este problema de optimización.

Para mitigar los riesgos de no cumplir con la demanda de mineral, debido a incertidumbres de las operaciones, los operadores de minas a menudo asignan más camiones de lo necesario. Este enfoque de aversión al riesgo conduce al uso ineficiente de camiones, lo que resulta en largas colas de camiones en los vertederos, en palas, o ambos. Su asignación, aun cuando no sean necesarios, deja menos camiones para otras tareas, como el transporte de desecho, que a veces es crítico. En respuesta, los operarios mineros suelen resolver la escasez de camiones a corto plazo mediante el alquiler de otros vehículos, pero esta resolución es costosa (Ta, Ingolfsson y Doucette, 2013).

Por lo general, los despachadores de camiones programan la asignación al comienzo en turnos de 12 horas, basados en datos y experiencias del usuario. Dependiendo de qué tanta experiencia haya tenido el encargado de la asignación, se puede esperar una mayor o menor eficiencia. Asegurar que los despachadores tengan las habilidades requeridas para la asignación eficiente de camiones requiere un proceso completo de capacitación, pero esa capacitación no garantiza la coherencia u optimización en la asignación de camiones. En relación a lo anterior, se ha estimado que el costo del transporte en las minas de tajo abierto representa hasta el $60 \%$ de los costos totales de extracción; por otro lado, el $32 \%$ de la energía total utilizada en las minas es consumida por las operaciones de transporte (Zhang y Xia, 2015; Sahoo, Bandyopadhyay y Banerjee, 2014). De manera similar, en minas subterráneas, las operaciones de carga, acarreo y descarga consumen hasta el $26 \%$ de la energía (LCG Energy Management Group, 2009).

Debido a la escala de operaciones en la minería, incluso una pequeña mejora en la eficiencia operativa se traduce en ahorros sustanciales durante las operaciones en cualquier tipo de 
mina (Burt et al., 2005). Por esto es importante trabajar profundamente en la planificación de las operaciones por las ventajas que se obtendrán gracias a una buena utilización de los recursos. El resultado de una inadecuada planificación conlleva a tener sobrecostos innecesarios que reducen las ganancias, generan una disminución de la ventaja competitiva y, finalmente, terminan en la pérdida de oportunidades. El modelo de costos de la operación minera es bastante complejo, puesto que agrupa una serie de parámetros, basado en esto, la utilización de un modelo matemático es necesario para calcular el número óptimo de camiones y determinar la mejor asignación.

\section{Contexto}

Se han utilizado diversos modelos de programación matemática para la optimización de recursos en la industria minera. Esta optimización consiste en la determinación óptima del número de camiones que se deben usar para el transporte del mineral. El desarrollo de una solución pertinente, ante esta clase de problemática, necesita de la identificación y del modelamiento de la agrupación de restricciones y variables que afectan directamente el proceso, esto con el objetivo de que se pueda optimizar la asignación de los recursos (Crovo, Martín y Rojas, 2007).

Según Alvarado (2009), en cualquier situación en donde participen una cantidad determinada de variables no negativas que, además, puedan vincularse entre sí, a través de relaciones de igualdad o de desigualdad, y que evidencien las restricciones del contexto con el objetivo de optimizar una operación, puede ser desarrollado a través de un modelo de programación matemática. Asimismo, si las variables y las restricciones relacionadas al modelo se definen mediante proposiciones lineales, se puede determinar como un modelo de programación lineal.

Según Zhang y Xia (2015), la gestión de la flota de camiones se modela como un problema de programación lineal entera, que tiene como objetivo cumplir la meta de producción con un costo operativo mínimo. Este autor enfoca el problema de tal manera que su modelo esté compuesto por la determinación del número de viajes, desde una zona de extracción a un sitio de descarga, considerando la duración de la jornada de trabajo, de modo que la producción objetivo sea alcanzada y el costo de operación del camión se reduzca al mínimo. Como el costo de operación del camión está directamente relacionado con la cantidad de camiones utilizados, siempre es deseable usar la menor cantidad de camiones para terminar el trabajo de transporte requerido, por tal motivo la investigación también se enfoca en identificar el tamaño óptimo de la flota de camiones.

Otra investigación relacionada es la de Temeng, Francis y Frendewey (1997), quienes adicionalmente a buscar la optimización de la flota de camiones, también se enfocaron en la optimización de las rutas de viaje, pues estas están directamente relacionadas con el tiempo 
de viaje desde los puntos de carga, a través de las palas, hasta las zonas de descarga. La maximización de la producción se logra utilizando las rutas de viaje más rápidas en tiempo tanto como sea posible. Los autores encaminan su modelo matemático y su algoritmo de solución para tener respuestas rápidas a cambios inesperados. De acuerdo a lo mencionado, formulan un algoritmo utilizando programación lineal que determina el numero óptimo de palas, el numero óptimo de camiones, los criterios de asignación de camiones a las palas y un modelo de transporte para la optimización de rutas.

Por otra parte, los autores Ta, Kresta, Forbes y Marquez (2005) desarrollan un modelo de asignación de camiones a través de una programación estocástica, dado que los objetivos de esta solución dependen de variables complejas como la configuración física de la mina, las condiciones de operación, la economía u objetivos de gestión y la cultura de la empresa. Los autores indican que, normalmente, en el desarrollo de los algoritmos matemáticos, utilizando programación lineal, se basan en parámetros con valores promediados, como, por ejemplo, la carga del camión y el tiempo de ciclo del camión, que a su vez están sujetos de otros parámetros con valores promediados como tiempo de carga, tiempo de descarga, tiempo de viaje de ida lleno, tiempo de viaje de regreso vacío, etc. La utilización de una programación estocástica se ve beneficiada siempre y cuando se trabaje con parámetros con valores inciertos y de corte aleatorio.

En la investigación realizada por Upadhyay y Askari-Nasab (2016), en una operación minera de hierro a tajo abierto en el sur de Irán, se presenta otro caso de optimización en la asignación de camiones, pero asociado a las palas. Los autores buscan maximizar la producción, minimizar las desviaciones respecto al tonelaje y a la cantidad de mineral que se suministra a cada planta (con respecto al esperado) y minimizar el costo operativo. Para ello, plantean la utilización de la programación lineal entera mixta, pero haciendo uso de un enfoque basado en la programación por objetivos (dado la cantidad de problemas que buscan abordar a la vez). Las variables que consideran son: las palas, los bancos de extracción, una flota heterogénea de camiones, los tipos de material (mineral y desmonte) y los destinos (plantas concentradoras, stockpiles y botaderos). Según explican, el modelo cumplió con los objetivos propuestos, elevando la utilización promedio de plantas, camiones y palas (en $99 \%$, en $92 \%$ y en $95 \%$, respectivamente).

Un estudio realizado en la unidad de Kelvin, sobre una operación minera subterránea de cobre, por los investigadores Nehring, Topal y Knights (2010), muestra como, al utilizar la programación lineal entera mixta, se puede minimizar el uso del equipamiento operativo en interior de mina, compuesto por camiones y cargadores frontales de bajo perfil, para darle cumplimiento a la producción objetiva establecida. En este estudio, se revisa la particularidad del acarreo minero en operaciones de socavón, donde se utilizan los camiones para movimientos de mineral por encima de $1 \mathrm{~km}$ y los cargadores frontales de bajo perfil para distancias menores. En el desarrollo del modelo matemático utilizan las siguientes variables: una flota de camiones y de cargadores frontales de bajo perfil, la producción objetivo y las distancias de traslado de mineral. Según 
los resultados obtenidos se confirmó una evidente optimización en el uso de equipamiento de acarreo sobre las prácticas de asignación manual que se venían realizando.

El uso de la técnica matemática de programación lineal también fue empleado en el trabajo realizado por Bajany, Xia y Zhang (2017) en una operación minera de tajo abierto. En ese trabajo los autores buscaban minimizar el uso de combustible de una flota de camiones heterogénea, cumpliendo con la demanda de los sitios de descarga. Teniendo en cuenta esto, lo que su modelo determina es la cantidad óptima de viajes que cada camión realiza en cada ruta de la mina, teniendo en cuenta variables como capacidad, velocidad cuando están vacíos y velocidad cuando están cargados; la capacidad, consumo de combustible cuando están inactivos y el consumo de combustible cuando están en operación de las palas y la topografía de la mina (distancias entre las palas y los sitios de descarga, demanda por hora de los sitios de descarga). Tal como presentan en su investigación, la solución de esta investigación muestra una notoria disminución en el consumo de combustible de los camiones y de las palas $(8,82 \%$ y 4,49\%, respectivamente), logrando así un ahorro de combustible total de 4,64 \%.

Según la literatura revisada, y como lo sostiene Elbrond (1979), la solución más adecuada para la optimización de recursos mineros (por ejemplo, la flota de camiones) es la simulación completa de todo el proceso y la utilización de procedimientos de optimización eficientes (por ejemplo, la programación lineal).

\section{Metodología}

\subsection{Programación lineal}

Según Stroud (2003), la programación lineal se define como "un método matemático para resolver un problema de optimización en donde la función objetivo es lineal y las restricciones son ecuaciones lineales o desigualdades lineales". Aunque la programación lineal es aplicable en una amplia variedad de contextos, ha sido desarrollada y aplicada con más frecuencia en las decisiones relacionadas con producción (Salvatore y Brooker, 2015).

Con el fin de aplicar la programación lineal en operaciones específicas, es necesario formular un modelo matemático. El término "formulación" se refiere al proceso de convertir las descripciones verbales y los datos numéricos en expresiones matemáticas, que representan relaciones entre las variables de decisión, la función objetivo y las restricciones (Lawrence, 2017).

Una vez formulado el problema como un programa lineal, se pueden usar herramientas computacionales como STORM, TORA, QSB, LINDO, etc., para solucionar el problema. En este sentido, la resolución del modelo es relativamente sencilla. La parte más difícil de aplicar esta metodología es formular el problema e interpretar la solución (McDill, 1999). 
En el campo de optimización, el significado del término "solución" no es necesariamente la respuesta final de un problema. Derigs (2009) explica que, dentro de este ámbito, cualquier especificación de valores para las variables de decisión se denomina una solución, independientemente de si es o no una opción deseable -o incluso permisible. Al resultado que satisface todas las restricciones se le conoce como factible, pero si además cuenta con el valor más favorable en la función objetivo, adquiere el nombre de solución óptima.

Diversos escritos sobre esta materia (Taha, 2004; Matousek y Gärtner, 2007; Bazaraa, Jarvis y Sherali, 2011) coinciden en que un modelo basado en un programa lineal está compuesto por un conjunto de variables de decisión, una función objetivo lineal que indica la contribución de cada variable al resultado deseado y un grupo de restricciones lineales que describen los límites en los valores de las variables.

A continuación se presenta la teoría fundamental de la programación lineal, la cual es universal y fue extraída de los escritos mencionados.

\subsubsection{Ecuaciones lineales}

Todas las ecuaciones y desigualdades en un programa lineal deben, por definición, ser lineales.

Una función lineal tiene la siguiente forma (1):

$$
a 0+a 1 \times 1+a 2 x 2+a 3 x 3+\cdots+a n x n=0
$$

(1) Forma de una función lineal

En general, las a son los llamados coeficientes de la ecuación; también conocidos como parámetros. Lo importante de los coeficientes es que son valores fijos, basados en la naturaleza subyacente del problema que se está resolviendo. Las $x$ son las variables de la ecuación; se les permite tomar un rango de valores dentro de los límites definidos por las restricciones.

\subsubsection{Las variables de decisión}

Las variables en la programación lineal son un conjunto de valores que deben determinarse para resolver el problema; es decir, el problema se resuelve cuando se han identificado los mejores totales de las variables. Típicamente, las variables representan la cantidad de un recurso a usar o el nivel de alguna actividad. 


\subsubsection{La función objetivo}

El objetivo de un problema de programación lineal será maximizar o minimizar algún valor numérico. La función objetivo indica cómo cada variable contribuye al valor que se optimizará para resolver el problema.

La función objetivo toma la siguiente forma general (2):

$$
\text { maximizar o minimizar } Z=\sum_{i=1}^{n} c_{i} X_{i}
$$

(2) Forma de la función objetivo

En donde $c_{i}=$ coeficiente de la función objetivo correspondiente a la inésima variable y $X_{i}=i^{\text {nésima }}$ variable de decisión.

Los coeficientes de la función objetivo indican la contribución al valor de la función objetivo de una unidad de la variable correspondiente.

\subsubsection{Las restricciones}

Las restricciones definen los valores posibles que pueden tomar las variables de un problema de programación lineal. Típicamente representan restricciones de recursos, o el nivel mínimo o máximo de alguna actividad o condición.

Toman la siguiente forma general (3):

$$
\text { Sujeto } a \sum_{i=1}^{n} a_{i j} X_{i} \leq b_{j} \quad j=1,2, \ldots, m
$$

(3) Forma general de una programación lineal

en donde, $X_{i}=i^{\text {nésima }}$ variable de decisión

$a_{i j}=$ al coeficiente en en la restricción $j$ y

$b_{j}=$ el coeficiente del lado derecho en la restricción $j$

Según Sanders y Reid (2012), para considerar a un programa lineal como tal, este debe contar con las suposiciones implícitas de proporcionalidad, aditividad, divisibilidad y certeza. Estos se explican a continuación: 
- Proporcionalidad: la contribución de las variables de decisión y de las restricciones en la función objetivo es proporcional a su valor. Por ejemplo, si se duplica el valor de una variable, también se duplicará la contribución de esa variable a la función objetivo y cada restricción en la cual aparece esa variable.

- Aditividad: el valor total de la función objetivo y cada función de restricción se obtienen sumando los aportes individuales de cada variable.

- Divisibilidad: las variables de decisión pueden tomar cualquier valor numérico real dentro de algún rango especificado por las restricciones. Es decir, las variables no están restringidas a valores enteros.

- Certeza: la solución óptima obtenida para un problema específico siempre será inmejorable. No obstante, si los valores de los parámetros son erróneos, entonces, la solución resultante lo será también.

\subsection{Programación por objetivos}

Como se ha mostrado, el propósito de los modelos basados en la programación lineal es resolver un único objetivo. Sin embargo, a partir de la práctica industrial y financiera moderna, está comprobado que maximizar las utilidades no necesariamente es el único objetivo que se busca alcanzar.

Charnes y Stedry (1966) no encuentran evidencia de que las empresas, efectivamente, construyan funciones focalizadas en maximizar la utilidad a largo plazo; además, sugieren que "los supuestos beneficios de la maximización de la utilidad no son operativos (incluso) si, lógica o tautológicamente, se puede demostrar que son válidos".

En las operaciones de planificación a largo plazo es mucho más habitual que los administradores hagan proyecciones que abarquen toda una gama de indicadores e intenten organizar una estrategia coherente para cumplir con estas proyecciones deseadas (Kornbluth, 1973).

Esto sería el caso habitual de la administración de las corporaciones en Estados Unidos, quienes se enfocan en una variedad de objetivos, por ejemplo, mantener ganancias estables, aumentar (o mantener) la cuota de mercado, diversificar productos, mantener precios estables, mejorar la moral del trabajador, mantener el control familiar del negocio y aumentar el prestigio de la compañía (Hillier y Lieberman, 2014). En síntesis, un proceso de toma de decisión no suele acotarse al cumplimiento de un único objetivo, de hecho, las decisiones más importantes suelen surgir en una atmósfera de confrontación en donde diferentes intereses se contraponen para llegar a una solución (Tamiz, Jones y Romero, 1998). 
Los métodos de programación de objetivos amplían nuestras capacidades de modelado al ofrecer formas de tratar con más de un objetivo a la vez. La programación por objetivos se puede entender como una extensión o generalización de la programación lineal para manejar múltiples medidas objetivas, normalmente en conflicto. A cada una de estas medidas se le asigna un objetivo o valor objetivo que se debe alcanzar. Las desviaciones no deseadas de este conjunto de valores objetivo se minimizan en una función de logro. Esto puede ser un vector o una suma ponderada, dependiendo de la variante de programación de objetivos utilizada.

\section{Caso de investigación}

\subsection{Datos de entrada}

Para este trabajo, se han considerado datos de una empresa minera peruana productora de concentrados de zinc $(\mathrm{Zn})$, plomo $(\mathrm{Pb})$, plata $(\mathrm{Ag})$ y cobre $(\mathrm{Cu})$. Estos minerales los obtiene de seis minas subterráneas: San Cristóbal, Carahuacra, Andaychagua, Ticlio, Animón e Islay. Estos minerales son procesados en cuatro plantas concentradoras: Victoria, Andaychagua, Mahr Tunel y Animón.

Cada una de las minas subterráneas tiene una ley particular sobre los cuatro minerales indicados previamente. La concentración de estos minerales (ley de mineral) por tonelada es cambiante con el tiempo, debido a la geografía de la zona, la ubicación de las vetas y al avance de la operación realizada en cada mina (tabla 1).

Tabla 1

Leyes de los minerales

\begin{tabular}{lcccc}
\hline Minas subterráneas & $\begin{array}{c}\text { Porcentaje } \\
\mathrm{Zn}\end{array}$ & $\begin{array}{c}\text { Porcentaje } \\
\mathrm{Pb}\end{array}$ & $\begin{array}{c}\text { Porcentaje } \\
\mathrm{Cu}\end{array}$ & $\begin{array}{c}\mathrm{Ag} \\
\text { 0z/TM }\end{array}$ \\
\hline San Cristóbal & 6,39 & 1,05 & 0,20 & 4,28 \\
\hline Carahuacra & 6,28 & 0,50 & 0,09 & 2,50 \\
\hline Andaychagua & 3,84 & 0,66 & 0,10 & 3,68 \\
\hline Ticlio & 6,07 & 1,63 & 0,19 & 1,60 \\
\hline Animón & 5,65 & 1,59 & 0,15 & 1,90 \\
\hline Islay & 1,89 & 0,83 & 0,05 & 7,15 \\
\hline
\end{tabular}

Elaboración propia

Dada la ubicación de las minas subterráneas y las plantas concentradoras, existe una relación mina-planta. Esto quiere decir que la extracción de minerales de una mina solo se puede tratar en la planta que tiene asignada (figura 1). 


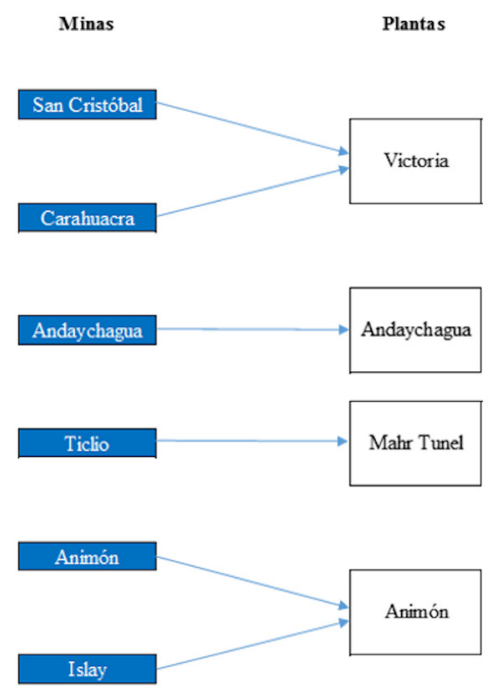

Figura 1. Relación mina-planta

Elaboración propia

Para la extracción del mineral, desde la zona de operación en interior de mina hasta la planta concentradora, se utilizan camiones con capacidad de 20 toneladas. El tipo de camiones es homogéneo en todas las unidades mineras debido a las dimensiones de los túneles de las minas. Sus camiones son de mayor capacidad para que puedan trabajar dadas las características de sus minas subterráneas.

El tiempo de viaje, desde la zona de carguío en interior de mina hasta las plantas concentradoras correspondientes, es variable y depende de la profundidad de la zona de operación subterránea, de las vías de transporte hacia superficie y de las distancias de las bocaminas hacia las plantas concentradoras. Los tiempos promedios, aproximados, de ida y vuelta de un camión desde la mina hasta la planta son los expresados en la tabla 2.

\section{Tabla 2}

Tiempos de viaje mina-planta promedio

\begin{tabular}{lll}
\hline Minas subterráneas & Planta concentradora & Tiempo viaje promedio (h) \\
\hline San Cristóbal & Victoria & 2,50 \\
\hline Carahuacra & & 1,50 \\
\hline Andaychagua & Andaychagua & 1,50 \\
\hline Ticlio & Mahr Tunel & 3,00 \\
\hline Animón & Animón & 1,50 \\
\hline Islay & & 2,50 \\
\hline
\end{tabular}

Elaboración propia 
Independientemente del tipo de mina, estas deben cumplir con un tonelaje de extracción mínimo diario, básicamente, para cubrir los costos operativos esenciales de cada mina (bombeo, ventilación y energía eléctrica). Adicionalmente, la empresa debe cumplir con producción objetivo mínima por cada mineral para poder cumplir los acuerdos contractuales pactados con sus clientes.

Cada mina subterránea tiene costos de extracción individuales debido a las características propias de cada una. Entre las variables que afectan estos costos se tienen: la topografía de la mina, el grado de sostenibilidad, el costo de los servicios auxiliares (bombeo, ventilación y energía eléctrica), la perforación, los explosivos, el equipamiento minero y su respectivo mantenimiento, la conservación de vías, el acarreo, etc.

Estos costos unitarios se obtienen sumando todos los costos que se tienen en la extracción del mineral, dividiéndolo por el número de toneladas extraídas en un determinado período de tiempo. En cuanto a las plantas concentradoras, estas son plantas de procesamiento de mineral donde se trata lo extraído y se busca separar el material inerte, elevando la concentración de los minerales a tratar (zinc, plomo, plata y cobre). Debido a la infraestructura de estas plantas, hay una capacidad máxima de tratamiento de mineral por cada una de ellas, la cual se refleja en la tabla 3.

\section{Tabla 3}

Capacidad máxima de la planta

\begin{tabular}{lc}
\hline Planta concentradora & Capacidad máxima planta (TM) \\
\hline Victoria & 5200 \\
\hline Andaychagua & 3450 \\
\hline Mahr Tunel & 2750 \\
\hline Animón & 5500 \\
\hline
\end{tabular}

Elaboración propia

De manera similar a lo que sucedía con las minas, cada planta concentradora tiene costos de tratamiento individuales debido a las características propias de cada una. Entre las variables que afectan estos costos se tienen: chancado, molienda, reactivos, flotación, filtrado, energía eléctrica, tratamiento de relave, relaveras, etc. Estos costos unitarios se obtienen sumando todos los costos que se tienen para el tratamiento del mineral, dividiéndolo por el número de toneladas tratadas en un determinado período de tiempo. Como se podrá apreciar, es la capacidad de tratamiento de las plantas la que determina los límites de extracción del mineral de cada una de las minas. 


\subsection{Modelo matemático}

El modelo planteando tiene como objetivo minimizar un puntaje basado en las desviaciones de tres objetivos. Los objetivos están relacionados al uso de la capacidad de las plantas, a la producción de los minerales y al costo total de la operación. A cada objetivo se le es asignado un peso (este dependiendo de su importancia relativa). Luego, este valor es multiplicado por la desviación del resultado del objetivo planteado. Mientras menor sea la desviación, menor será el puntaje, lo cual significa una mayor precisión en el cumplimiento de los objetivos.

a) índices

$i=\operatorname{mina} i=1,2, \ldots, x$

$j=$ mineral $j=1,2, \ldots, y$

$k=$ planta concentradora $k=1,2, \ldots, z$

en donde:

$x=$ número de minas

$y=$ número de minerales

$z=$ número de plantas concentradoras

b) Variable de decisión

cant $_{i}=$ cantidad de camiones asignados a la mina $i$

c) Parámetros

ley $_{i j}=$ ley del mineral $j$ en la mina $i$

precio $_{j}=$ precio del mineral $j$ en el mercado internacional

$\operatorname{carg}=$ capacidad de transporte de un camión

$t_{i}=$ tiempo promedio de viaje desde la mina $i$ hasta su respectiva planta

$c t r_{k}=$ costo de tratamiento (US\$/TM) de la planta $k$

cext $_{i}=$ costo de extracción (US\$/TM) de la mina $i$

capMAX $X_{k}=$ capacidad máxima de tratamiento de la planta $k$

$\operatorname{costMAX}=$ presupuesto límite establecido

$\operatorname{prodMin}_{j}=$ producción mínima mineral $j$

$r l c n_{i k}=$ relación de paridad entre la mina $i$ y la planta $k$

$\{1:$ en caso el mineral extraido de mina $i$ se trabaje en la planta $k$

0: caso contrario 
$W g h t_{k}=$ peso de penalización relacionado a la capacidad de utilización objetivo de la planta $k$

$W g h t_{j}=$ peso de penalización relacionado a la cantidad de concentrado objetivo del mineral $j$

WghtCost $=$ peso de penalización relacionado al costo total objetivo

$\operatorname{DesvCap}_{k}=$ desviación de la capacidad de utilización objetivo de la planta $k$

$\operatorname{DesvMin}_{j}=$ desviación de la cantidad de concentrado objetivo del mineral $j$

DesvCost $=$ desviación del costo total objetivo

d) Función objetivo

La función objetivo se muestra a continuación:

$P T=\left(\operatorname{DesvCap}_{k} \times W g h t_{k}\right)+\left(\operatorname{DesvMin}_{j} \times W g h t_{j}\right)+($ DesvCost $\times W g h t$ Cost $)$

(4) Función objetivo propuesta

e) Restricciones

$\operatorname{ObjCap}_{k}-\sum_{i=1}^{x} \operatorname{rlcn}_{i k}\left(\operatorname{carg} \times \operatorname{cant}_{i} \frac{24}{t_{i}}\right)=\operatorname{DesvCap}_{k} \quad k=1,2, \ldots, z$

(5) Restricción 1

Para determinar la desviación de la capacidad de utilización de una planta, primero, se debe precisar cuánto es la cantidad de extracción que será trasladada hacia esta planta. Ese monto se calcula multiplicando la carga de un camión por la cantidad de camiones asignados a las minas que depositan en dicha planta. Hallado el monto, este es restado del objetivo, dando lugar a la desviación.

$$
\operatorname{ObjMin}_{j}-\sum_{i=1}^{x} \operatorname{ley}_{i j}\left(\operatorname{carg} \times \operatorname{cant}_{i} \frac{24}{t_{i}}\right)=\operatorname{DesvMin}_{j} \quad j=1,2, \ldots
$$

\section{(6) Restricción 2}

La desviación de la cantidad de concentrado objetivo es hallada restando el monto concentrado total del objetivo. Para calcular el total de concentrado adquirido de un mineral, basta con hacer una sumatoria de la multiplicación de la ley de ese mineral por la cantidad de extracción en cada mina. 


$$
\text { ObjCost }-\sum_{i=1}^{x} \sum_{j=1}^{k} \operatorname{rlcn}_{i k}\left(\operatorname{carg} \times \operatorname{cant}_{i} \frac{24}{t_{i}}\right)\left(\operatorname{ctr}_{k}+\operatorname{cext}_{i}\right)=\text { DesvCost }
$$

(7) Restricción 3

El costo total de la operación se halla sumando los costos de extracción y los de tratamiento. El costo de extracción varía de mina a mina y el costo de tratamiento varía dependiendo de la planta. El costo total es directamente proporcional a la cantidad de extracción de cada mina, mientras mayor sea la extracción mayor será el costo total. De manera similar a las operaciones en la ecuaciones (6) y (7), para hallar la desviación del costo total objetivo, al costo total objetivo se le resta el costo total de la operación.

$$
\sum_{i=1}^{x} \operatorname{rlcn}_{i k}\left(\operatorname{carg} \times \operatorname{cant}_{i} \frac{24}{t_{i}}\right) \leq \operatorname{capMAX}_{k} \quad k=1,2, \ldots, z
$$

(8) Restricción 4

La cantidad de extracción que se dirige a cada planta debe de ser menor o igual a la capacidad máxima de tratamiento de dicha planta.

$$
\sum_{i=1}^{x} \operatorname{ley}_{i j}\left(\operatorname{carg} \times \operatorname{cant}_{i} \frac{24}{t_{i}}\right) \geq \operatorname{prodMin}_{j} \quad j=1,2, \ldots, y
$$

(9) Restricción 5

La cantidad de extracción de cada mineral debe de ser mayor o igual a la producción mínima establecida.

$$
\sum_{i=1}^{x} \operatorname{ley}_{i j}\left(\operatorname{carg} \times \operatorname{cant}_{i} \frac{24}{t_{i}}\right) \geq \operatorname{prodMin}_{j} \quad j=1,2, \ldots, y
$$

(10) Restricción 6

$$
x_{i} \geq 0, \quad x_{i} \in \mathbb{N}
$$

\section{(11) Restricción 7}

La cantidad de camiones a asignar a cada mina debe ser un número natural mayor o igual a 0 . 


\subsection{Experimentación y hallazgos}

Para la muestra de la experimentación, se utilizarán seis casos de prueba que representan diferentes situaciones a las que una empresa minera podría enfrentarse. Cabe mencionar que, para facilitar el análisis y la interpretación de los resultados, se mantendrá una misma distribución de pesos por cada objetivo y en cada una de las ejecuciones. Los casos de prueba en sí están dirigidos a probar escenarios que van más allá de los límites de la operación normal, esto con el fin de medir la solidez del sistema y el manejo de errores.

La construcción de los casos de prueba fue hecha con la ayuda de un grupo de usuarios finales, de la misma minera, que proporcionó los datos de entrada. Tal y como sugiere la literatura, esto es bastante beneficioso, porque puede aprovecharse el dominio y la experiencia de estas personas para establecer escenarios más acordes a la realidad. Los casos de prueba variarán según los valores que se le asigna a cada objetivo, manteniendo los datos paramétricos constantes.

Cabe mencionar que los pesos establecidos para cada objetivo fueron determinados según un análisis exhaustivo hecho por los usuarios finales. El análisis se basó en data histórica de la empresa y en sus futuros planes y convicciones. Considerando lo anterior, el método empleado para la experimentación será una mezcla entre el uso de Test Bases y el de Real-time and Historic Data Sets. Estos métodos conformarán el proceso de validación. Asimismo, para ejecutar el modelo se utilizó un complemento de Microsoft Excel llamado Solver. El motivo fue, básicamente, por la flexibilidad durante la diagramación del modelo en sí que ofrece esta herramienta.

Para el primer caso de prueba, consideraremos una situación en donde la combinación de objetivos son valores pequeños, pero con un presupuesto amplio (tabla 4).

Tabla 4

Objetivos del caso de prueba 1

\begin{tabular}{cccc}
\hline Variable & Objetivos & Peso por 1\% de desviación & 1\% del objetivo \\
\hline VIC- & 5200 & 3 & 52 \\
\hline AND- & 3450 & 3 & 34,5 \\
\hline MAH- & 2750 & 3 & 27,5 \\
\hline ANI- & 5500 & 3 & 55 \\
\hline ZN+ & 500 & 10 & 5 \\
\hline PB+ & 100 & 10 & 1 \\
\hline CU+ & 15 & 10 & 0,15 \\
\hline Ag+ & 10000 & 10 & 100 \\
\hline DOL+ & 2000000 & -5 & 20000 \\
\hline
\end{tabular}

Elaboración propia 
Al ejecutar el sistema obtenemos los resultados dados en la tabla 5.

Tabla 5

Resultados del caso de prueba 1

\begin{tabular}{lrrr}
\hline Variable & Objetivos & Desviación & Puntaje \\
\hline VIC- & 2304,00 & 2896,00 & 167,08 \\
\hline AND- & 2560,00 & 890,00 & 77,39 \\
\hline MAH- & 2720,00 & 30,00 & 3,27 \\
\hline ANI- & 2304,00 & 3196,00 & 174,33 \\
\hline ZN+ & 502,71 & 2,71 & 5,41 \\
\hline PB+ & 111,42 & 11,42 & 114,18 \\
\hline CU+ & 15,06 & 0,06 & 3,95 \\
\hline Ag+ & 10044,27 & 44,27 & 4,43 \\
\hline DOL+ & 1212480,00 & 787520,00 & $-196,88$ \\
\hline Puntaje & & & 353,15 \\
\hline
\end{tabular}

\begin{tabular}{lc}
\hline $\begin{array}{l}\text { Minas } \\
\text { subterráneas }\end{array}$ & $\begin{array}{c}\text { Mejor arreglo } \\
\text { de camiones }\end{array}$ \\
\hline San Cristóbal & 12 \\
\hline Carahuacra & 0 \\
\hline Andaychagua & 8 \\
\hline Ticlio & 17 \\
\hline Animón & 0 \\
\hline Islay & 12 \\
\hline Total & 49 \\
\hline
\end{tabular}

Elaboración propia

Como se ve en la tabla 5, el sistema no utiliza las minas Carahuacra y Animón para cumplir con los objetivos planteados. Con esto evidenciamos que el modelo es capaz de determinar cuándo es o no necesario extraer de una mina. Si bien existen programaciones que cumplirían con los objetivos utilizando todas las minas, el porqué el sistema decide no utilizarlo es debido a la menor desviación de los objetivos que tiene utilizando esta programación.

Para el segundo caso de prueba, considerarnos los mismos objetivos que el caso anterior, pero ahora acotaremos el presupuesto que se tiene a $\$ 950000$ (tabla 6).

\section{Tabla 6}

Objetivos del caso de prueba 2

\begin{tabular}{lccc}
\hline Variable & Objetivos & Peso por 1\% de desviación & 1\% del objetivo \\
\hline VIC- & 5200 & 3 & 52 \\
\hline AND- & 3450 & 3 & 34,5 \\
\hline MAH- & 2750 & 3 & 27,5 \\
\hline ANI- & 5500 & 3 & 55 \\
\hline ZN+ & 500 & 10 & 5 \\
\hline & & & (continúa)
\end{tabular}


(continuación)

\begin{tabular}{lrrr}
\hline $\mathrm{PB}+$ & 100 & 10 & 1 \\
\hline $\mathrm{CU}+$ & 15 & 10 & 0,15 \\
\hline $\mathrm{Ag}+$ & 10000 & 10 & 100 \\
\hline $\mathrm{DOL}+$ & 950000 & -5 & 9500 \\
\hline
\end{tabular}

Elaboración propia

Al ejecutar el sistema obtenemos los resultados de la tabla 7.

Tabla 7

Resultados del caso de prueba 2

\begin{tabular}{lrrr}
\hline Variable & Objetivos & Desviación & Puntaje \\
\hline VIC- & 3264,00 & 1936,00 & 111,69 \\
\hline AND- & 0,00 & 3450,00 & 300,00 \\
\hline MAH- & 2720,00 & 30,00 & 3,27 \\
\hline ANI- & 2112,00 & 3388,00 & 184,80 \\
\hline ZN+ & 507,80 & 7,80 & 15,60 \\
\hline PB+ & 102,80 & 2,80 & 27,97 \\
\hline CU+ & 15,06 & 0,06 & 3,95 \\
\hline Ag+ & 10219,97 & 219,97 & 22,00 \\
\hline D0L+ & 911360,00 & 38640,00 & $-20,34$ \\
\hline Puntaje & & & 648,94 \\
\hline
\end{tabular}

\begin{tabular}{lc}
\hline $\begin{array}{l}\text { Minas } \\
\text { subterráneas }\end{array}$ & $\begin{array}{c}\text { Mejor arreglo } \\
\text { de camiones }\end{array}$ \\
\hline San Cristóbal & 7 \\
\hline Carahuacra & 6 \\
\hline Andaychagua & 0 \\
\hline Ticlio & 17 \\
\hline Animón & 6 \\
\hline Islay & 1 \\
\hline Total & 37 \\
\hline
\end{tabular}

Elaboración propia

Nótese que el sistema efectivamente puede abarcar la misma producción objetivo a un menor costo y al mismo tiempo con una significativa menor de cantidad de camiones. Sin embargo, si comparamos el puntaje en ambos casos, 353,15 y 648,94, el puntaje del segundo caso de prueba está cerca a ser el doble que el primero. Para el sistema esto significa que (aunque parezca contradictorio en efectos de lo que verdaderamente le convendría a la empresa), en el primer caso, se están cumpliendo de manera más adecuada los objetivos. Esto es causado porque las desviaciones de todas las variables son mucho más grandes en el segundo caso, puesto que esta es la única forma de cumplir con el presupuesto asignado.

Para el tercer y cuarto caso, se simularán contextos en los cuales, debido a eventos como el alza en el precio de algún mineral, se querrá extraer la mayor cantidad posible. Para esto se 
comenzará con una producción objetivo de plata de 22400 oz que, considerando las leyes y las capacidades productivas impuestas en el modelo, es un valor bastante alto (tabla 8).

Tabla 8

Objetivos del caso de prueba 3

\begin{tabular}{lrcc}
\hline Variable & Objetivos & $\begin{array}{c}\text { Peso por 1\% } \\
\text { de desviación }\end{array}$ & 1\% del objetivo \\
\hline VIC- & 5200 & 3 & 52 \\
\hline AND- & 3450 & 3 & 34,5 \\
\hline MAH- & 2750 & 3 & 27,5 \\
\hline ANI- & 5500 & 3 & 55 \\
\hline ZN+ & 500 & 10 & 5 \\
\hline PB+ & 100 & 10 & 1 \\
\hline CU+ & 15 & 10 & 0,15 \\
\hline Ag+ & 22400 & 10 & 224 \\
\hline DOL+ & 2000000 & -5 & 20000 \\
\hline
\end{tabular}

Elaboración propia

Al ejecutar el sistema obtenemos los siguientes resultados de la tabla 9.

Tabla 9

Resultados del caso de prueba 3

\begin{tabular}{lrrr}
\hline Variable & Objetivos & Desviación & Puntaje \\
\hline VIC- & 5184,00 & 16,00 & 0,92 \\
\hline AND- & 2560,00 & 890,00 & 77,39 \\
\hline MAH- & 0,00 & 2750,00 & 300,00 \\
\hline ANI- & 5376,00 & 124,00 & 6,76 \\
\hline ZN+ & 536,17 & 36,17 & 72,35 \\
\hline PB+ & 123,40 & 23,40 & 233,98 \\
\hline CU+ & 16,15 & 1,15 & 76,48 \\
\hline Ag+ & 22408,37 & 8,37 & 0,37 \\
\hline DOL+ & 1585600,00 & 414400,00 & $-103,60$ \\
\hline Puntaje & & & 664,66 \\
\hline
\end{tabular}

\begin{tabular}{lc}
\hline Minas subterráneas & $\begin{array}{c}\text { Mejor arreglo } \\
\text { de camiones }\end{array}$ \\
\hline San Cristóbal & 27 \\
\hline Carahuacra & 0 \\
\hline Andaychagua & 8 \\
\hline Ticlio & 0 \\
\hline Animón & 0 \\
\hline Islay & 28 \\
\hline Total & 63
\end{tabular}

Elaboración propia 
Los resultados están relacionados con los objetivos planteados. Fíjese que para el caso de prueba 3, el sistema solo usa 3 de las 6 minas: San Cristóbal, Andaychagua e Islay. Esto tiene bastante sentido, considerando que estas minas son las que tienen la mayor ley de plata, siendo 4,23, 3,17 y 7,42 oz/TM, respectivamente. Cabe resaltar el desuso de las otras minas. El sistema opta por no usarlas, ya que de lo contrario se podría incurrir en un sobrecumplimiento de las producciones objetivo, tanto de la plata como de los otros minerales, cosa que penaliza el puntaje.

Ahora obtendremos una producción de zinc. Al igual que en el caso anterior, probaremos con una producción objetivo alta, considerando las leyes y las capacidades productivas impuestas en el modelo, esta será de 998 TM (tabla 10).

Tabla 10

Objetivos del caso de prueba 4

\begin{tabular}{cccc}
\hline Variable & Objetivos & Peso por $1 \%$ de desviación & $1 \%$ del objetivo \\
\hline VIC- & 5200 & 3 & 52 \\
\hline AND- & 3450 & 3 & 34,5 \\
\hline MAH- & 2750 & 3 & 27,5 \\
\hline ANl- & 5500 & 3 & 55 \\
\hline ZN+ & 998 & 10 & 9,98 \\
\hline PB+ & 100 & 10 & 1 \\
\hline CU+ & 15 & 10 & 0,15 \\
\hline Ag+ & 10000 & 10 & 100 \\
\hline DOL+ & 2000000 & -5 & 20000 \\
\hline
\end{tabular}

Elaboración propia

Al ejecutar el sistema obtenemos los resultados de la tabla 11.

Tabla 11

Resultados del caso de prueba 4

\begin{tabular}{lrrc}
\hline Variable & Objetivos & Desviación & \\
\hline VIC- & 5184,00 & 16,00 & 0,92 \\
\hline AND- & 3200,00 & 250,00 & 21,74 \\
\hline MAH- & 2720,00 & 30,00 & 3,27 \\
\hline ANI- & 5440,00 & 60,00 & 3,27 \\
\hline
\end{tabular}

\begin{tabular}{lc}
\hline $\begin{array}{l}\text { Minas } \\
\text { subterráneas }\end{array}$ & $\begin{array}{c}\text { Mejor arreglo } \\
\text { de camiones }\end{array}$ \\
\hline San Cristóbal & 27 \\
\hline Carahuacra & 0 \\
\hline Andaychagua & 10 \\
\hline Ticlio & 17
\end{tabular}




\begin{tabular}{lrrr}
\hline $\mathrm{ZN}+$ & 998,24 & 0,24 & 0,24 \\
\hline $\mathrm{PB}+$ & 225,09 & 125,09 & 1250,88 \\
\hline $\mathrm{CU}+$ & 29,62 & 14,62 & 974,61 \\
\hline $\mathrm{Ag}+$ & 22186,98 & 12186,98 & 1218,70 \\
\hline $\mathrm{DOL}+$ & 1938240,00 & 61760,00 & $-15,44$ \\
\hline Puntaje & & & 3458,20 \\
\hline
\end{tabular}

\begin{tabular}{lc}
\hline Animón & 17 \\
\hline Islay & 0 \\
\hline Total & 71 \\
\hline
\end{tabular}

Elaboración propia

Como se puede apreciar en la tabla 11, el objetivo es completado satisfactoriamente. Sin embargo, comparando estos resultados con los del caso anterior, vemos que el costo al extraer una gran cantidad de zinc es mucho más alto que al extraer una gran cantidad de plata. Por otro lado, con respecto a la asignación de camiones, las minas en donde se extrae son, además, las más ricas en zinc: San Cristóbal con una ley de 6,45 \%, Ticlio con $7,44 \%$, Animón con $5,86 \%$ y Andaychagua con 4,46 \%. A simple vista parecería que hubiera un error, pues se le ha asignado menos camiones a Ticlio que a San Cristóbal, a pesar de tener una ley más grande de zinc. Esto no es un error; es causado por la restricción de la planta concentradora Mahr Tunel que, con una capacidad de tratamiento máximo de 2750 TM diaria, limita qué tanto se puede extraer de la mina Ticlio. La planta concentradora Victoria, con una capacidad de tratamiento máximo de 5200 TM diaria, tiene más potencial para abarcar grandes cantidades de extracción.

Asimismo, cabe señalar otro punto valioso, el porqué de la asignación de 0 camiones a la mina Carahuacra a pesar de tener una ley 5,37 \% de zinc, siendo inclusive mayor a la de Andaychagua. Esto se debe a que la extracción de la mina Carahuacra es enviada a la planta Victoria para su tratamiento, igual que la de la mina San Cristóbal. Considerando esto, el sistema indudablemente priorizará ocupar la totalidad de la planta solo con San Cristóbal, pues tiene una ley mayor a la de Carahuacra: 6,45 \% y 5,37 \%, respectivamente. Para los dos últimos casos, se experimentará una programación ideal, en otras palabras, es donde la producción objetivo busca los niveles más altos de cada uno de los minerales al menor costo posible. Comenzaremos con un caso imposible (tabla 12). 
Tabla 12

Objetivos del caso de prueba 5

\begin{tabular}{lrrr}
\hline Variable & Objetivos & Peso por $1 \%$ de desviación & $1 \%$ del objetivo \\
\hline VIC- & 5200 & 3 & 52 \\
\hline AND- & 3450 & 3 & 34,5 \\
\hline MAH- & 2750 & 3 & 27,5 \\
\hline ANI- & 5500 & 3 & 55 \\
\hline ZN+ & 895 & 10 & 8,95 \\
\hline PB+ & 195 & 10 & 1,95 \\
\hline CU+ & 24 & 10 & 0,2 \\
\hline Ag+ & 18600 & 10 & 186 \\
\hline DOL+ & 2000000 & -5 & 20000 \\
\hline
\end{tabular}

Elaboración propia

Al ejecutar el sistema, nos muestra el error de la figura 2.

\section{Solver no encontró ninguna solución viable.}

Solver no puede encontrar un punto en el que se cumplan todas las restricciones.

Figura 2. Error en el caso de prueba 5

Esto se origina porque las producciones objetivo que introducimos son excesivamente altas y, considerando las restricciones de la capacidad máxima de las plantas, no existe solución que permita cumplir con tales objetivos.

Ahora se considerarán objetivos posibles con altos objetivos de producción (tabla 13). 
Tabla 13

Objetivos del caso de prueba 6

\begin{tabular}{lrrr}
\hline Variable & Objetivos & Peso por $1 \%$ de desviación & 1\% del objetivo \\
\hline VIC- & 5200 & 3 & 52 \\
\hline AND- & 3450 & 3 & 34,5 \\
\hline MAH- & 2750 & 3 & 27,5 \\
\hline ANI- & 5500 & 3 & 55 \\
\hline ZN+ & 1000 & 10 & 10 \\
\hline PB+ & 225 & 10 & 2,25 \\
\hline CU+ & 29 & 10 & 0,29 \\
\hline Ag+ & 22180 & 10 & 221,8 \\
\hline DOL+ & 2000000 & -5 & 20000 \\
\hline
\end{tabular}

Elaboración propia

Al ejecutar el sistema obtenemos los resultados de la tabla 14.

Tabla 14

Objetivos del caso de prueba 6

\begin{tabular}{lrrr}
\hline Variable & Objetivos & Desviación & Puntaje \\
\hline VIC- & 5184,00 & 16,00 & 0,92 \\
\hline AND- & 3200,00 & 250,00 & 21,74 \\
\hline MAH- & 2720,00 & 30,00 & 3,27 \\
\hline ANI- & 5440,00 & 60,00 & 3,27 \\
\hline ZN+ & 896,29 & 1,29 & 1,44 \\
\hline PB+ & 195,71 & 0,71 & 3,65 \\
\hline CU+ & 24,82 & 0,82 & 34,13 \\
\hline Ag+ & 18603,87 & 3,87 & 0,21 \\
\hline DOL+ & 1880640,00 & 119360,00 & $-29,84$ \\
\hline Puntaje & & & 38,80 \\
\hline
\end{tabular}

\begin{tabular}{lc}
\hline $\begin{array}{l}\text { Minas } \\
\text { subterráneas }\end{array}$ & $\begin{array}{c}\text { Mejor arreglo } \\
\text { de camiones }\end{array}$ \\
\hline San Cristóbal & 17 \\
\hline Carahuacra & 6 \\
\hline Andaychagua & 10 \\
\hline Ticlio & 17 \\
\hline Animón & 11 \\
\hline Islay & 10 \\
\hline Total & 71 \\
\hline
\end{tabular}

Elaboración propia

Los resultados mostrados en la tabla 14, entonces, representarían el mejor escenario para la empresa. 


\subsection{Herramienta computacional utilizada}

Basado en el modelo matemático propuesto y con los datos proporcionados por la empresa minera, se desarrolló un prototipo de lo que sería la solución final de este trabajo. Para ello, se utilizó el complemento Solver de Microsoft Excel. El motivo por el cual se optó por utilizar esta herramienta sobre otras, como Lingo o Storm, fue básicamente por su flexibilidad durante la diagramación del modelo en sí. Asimismo, teniendo en cuenta que es en realidad el Excel el que invoca a este complemento, el uso de Solver se empodera por las características que trae el Excel como tal, como la capacidad de incluir gráficos o botones programables a través del uso de macros.

A continuación, la definición del modelo matemático propuesto realizado en Solver (figura 3).

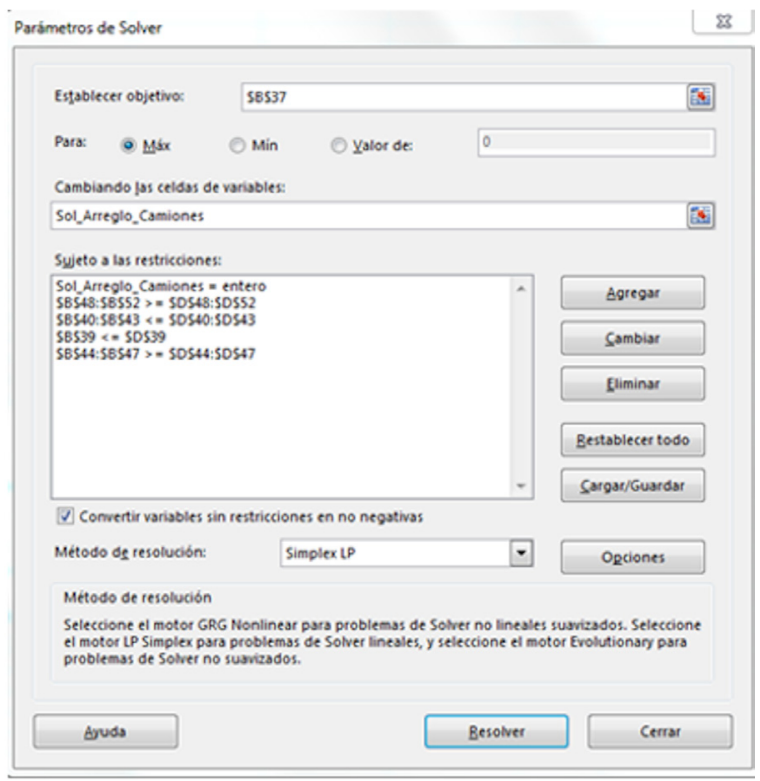

Figura 3. Modelo matemático definido en Solver

Elaboración propia

Como se puede apreciar en la figura 3, tanto las variables de decisión, las restricciones y la función objetivo hacen referencia a celdas de Excel. Esto se debe a que los datos que se ingresaron para tales partes del modelo están definidos en el mismo Excel. Cabe mencionar que si bien en la figura 17 no se encuentran todas las ecuaciones lineales definas previamente, esto es porque han sido introducidas indirectamente a través de fórmulas entre diferentes celdas de datos. 


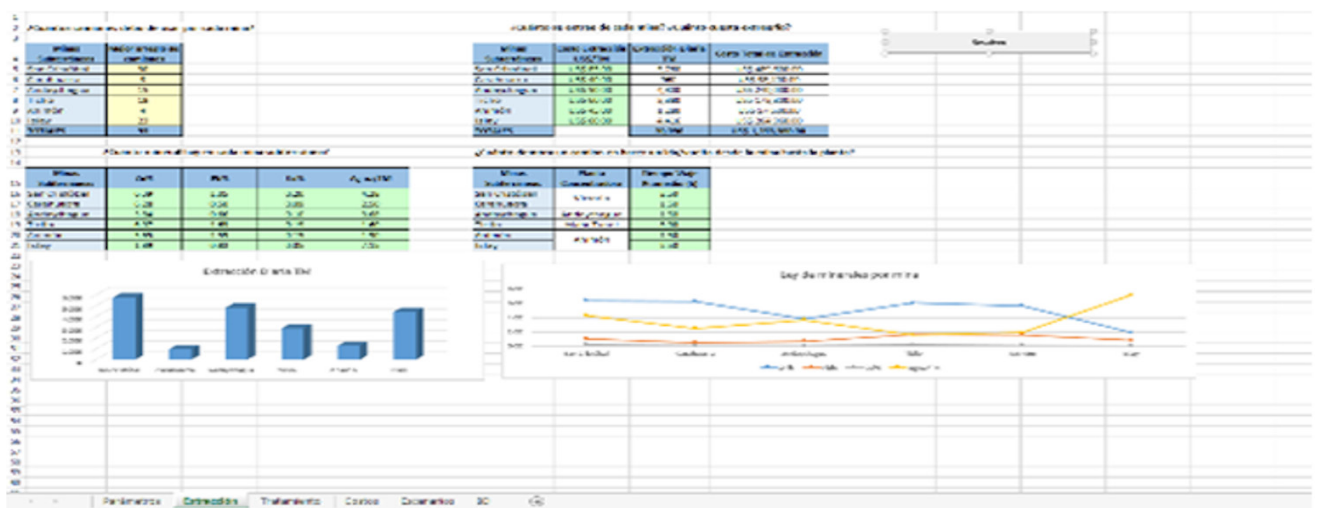

Figura 4. Vista previa del prototipo en Excel

Elaboración propia

El prototipo cuenta con cinco pestañas diferentes: "parámetros", básicamente donde se introducen todos los valores que funcionan como restricciones para el modelo; "extracción", pestaña en donde se da inicio a la ejecución del modelo, a través de Solver, y en donde guarda la solución. Además, almacena todo lo referente a la extracción como son las leyes de minerales, los tiempos de viaje promedio y los costos de extracción.

Por otra parte, la pestaña "tratamiento" guarda toda la data relacionada al proceso de tratamiento del mineral como son los costos de tratamiento por planta y la producción de concentrado por mineral de cada una. La de "costos" presenta los valores para hallar la utilidad total: total de ventas, costo de extracción y costo de tratamiento. Por último, las pestañas "escenarios" y "BD" son módulos adicionales que buscan simular las características que ya tendría el SSD, como es la comparación de escenarios y el almacenamiento periódico de las programaciones de camiones en una base de datos.

\section{Reflexión}

Podemos afirmar que el modelo funciona correctamente. Se logró la construcción de un modelo basado en la programación por objetivos que calcula el tamaño de la flota de camiones óptima y su adecuada asignación a las diferentes minas, con miras a minimizar las desviaciones de la utilización de la capacidad máxima de las plantas concentradoras y la producción de los minerales, al menor costo posible.

El uso de la programación por objetivos y la programación lineal son técnicas de investigación de operaciones bastante útiles y, tal y como se comprobó en otros estudios, particularmente, en cuanto a la asignación de camiones en el sector minero. Considerando la interpretación de los resultados, es indudablemente recomendable que la empresa minera utilice este modelo siempre 
que busque establecer objetivos relacionados a la producción de solo minerales, que, como grupo, tienen las leyes más altas. Esto debido a que podrían explotar de mejor manera el uso de sus instalaciones, generar mayores concentrados $y$, al mismo tiempo, minimizar el costo total.

\section{Futura continuación de la investigación}

Para darle continuidad a esta investigación, se recomienda investigar la inclusión de otras variables, tales como el uso de combustible, la cantidad de tiempo de transporte y la determinación de rutas entre minas y plantas. Tales factores, si bien no participan en el cumplimiento de los tres objetivos planteados para esta investigación, a través de una correcta medición y control podrían traer una mayor reducción en costos de la que el modelo planteado ofrece. Asimismo, añadir un nuevo conjunto de variables abriría paso a la inclusión de nuevos objetivos, por ejemplo, que la suma total de combustible de todos los camiones a utilizar no supere los $X$ litros.

\section{Referencias}

Alvarado Boirivant, J. (2009). La programación lineal aplicación de las pequeñas y medianas empresas. Reflexiones, 88(1), pp. 89-105.

Bajany, D. M., Xia, X., y Zhang, L. (2017). A MILP Model for Truck-shovel scheduling to minimize fuel consumption. Energy Procedia, 105, pp. 2739-2745.

Bazaraa, M. S., Jarvis, J. J., y Sherali, H. D. (2011). Linear programming and network flows. Hoboken, Estados Unidos: John Wiley \& Sons.

Burt, C., Caccetta, L., Hill, S., Welgama, P., Zerger, A., y Argent, R. M. (2005). Models for mining equipment selection. International Congress Modeling Simulation. Modelling and Simulation Society of Australia and New Zealand, pp. 1730-1736.

Charnes, A., y Stredy, A. (1966). The attainment of organizational goals through the appropriate selection of sub-unit goals. Operational Research and the Social Sciences. Tavistock Publications.

Crovo, A. S., Martín, C. O. y Rojas, L. P. (2007). Modelos de programación entera para un problema de programación de horarios para universidades. Revista Chilena de Ingeniería.

Derigs, U. (2009). Optimization and operations research (volume I). Oxford, Inglaterra: EOLSS Publications.

Elbrond, J., y Soumis, F. (1987). Towards integrated production planning and truck dispatching in open pit mines. International Journal of Surface Mining, pp. 1-6.

Hillier, F., y Lieberman, G. (2014). Introduction to operations research (10th ed.). New York: Mcgraw Hill Education. 
Kornbluth, J. S. H. (1973). A survey of goal programming. Omega, 1(2), pp. 193-205.

Lawrence, O. (2017). Linear programming as a tool for water resources management. International Journal of Constructive Research in Civil Engineering, 3(4), pp. 30-47.

LCG Energy Management Group. (2009). Investigation of current research related to the reduction of energy usage in mines through recycling, reuse, and other means.

Matousek, J., y Gärtner, B. (2007). Understanding and using linear programming. Berlín: Springer Science \& Business Media.

McDill, M. E. (1999). Forest Resource Management. Recuperado de https://www.courses.psu. edu/for/for466w_mem14/PDFs/

Nehring, M., Topal, E., y Knights, P. (2010). Dynamic short term production scheduling and machine allocation in underground mining using mathematical programming. Mining Technology, 119(4), pp. 212-220.

Sahoo, L. K., Bandyopadhyay, S., y Banerjee, R. (2014). Benchmarking energy consumption for dump trucks in mines. Applied Energy, 113, pp. 1382-1396.

Ta, C. H., Ingolfsson, A., y Doucette, J. (2013). A linear model for surface mining haul truck allocation incorporating shovel idle probabilities. European Journal of Operational Research, 231(3), pp. 770-778.

Ta, C. H., Kresta, J. V., Forbes, J. F., y Marquez, H. J. (2005). A stochastic optimization approach to mine truck allocation. International Journal of Mining, Reclamation and Environment, 19(3), pp. 162-175.

Taha, H. A. (2004). Investigación de operaciones. México: Pearson Educación.

Tamiz, M., Jones, D., y Romero, C. (1998). Goal programming for decision making: An overview of the current state-of-the-art. European Journal of Operational Research, 111(3), pp. 569-581.

Temeng, V. A., Francis, O. O., y Frendewey, Jr., J. O. (1997). Real-time truck dispatching using a transportation algorithm. International Journal of Surface Mining, Reclamation and Environment. 11(4), pp. 203-207.

Upadhyay, S. P., y Askari-Nasab, H. (2016). Truck-shovel allocation optimisation: a goal programming approach. Mining Technology, 125(2), pp. 82-92.

Zhang, L., y Xia, X. (2015). An integer programming approach for truck-shovel dispatching problem in open-pit mines. Energy Procedia, 75, pp. 1779-1784. 